\title{
From Clinical Practice Guideline to Clinical Pathway - Issues of Reference Model-Based Approach
}

\author{
Hannes Schlieter and Werner Esswein \\ Department of Business Management and Economics, Chair for Information Systems, \\ esp. ystems Engineering, Münchner Platz 3, 01062 Dresden, Germany \\ \{Hannes.Schlieter, Werner.Esswein\} atu-dresden.de
}

\begin{abstract}
Health care systems are facing new challenges such as the aging population, the increasing amount of chronicle diseases and the rapid development of medical technologies. The increasing of the related health care costs lead to the limits of affordability of health care systems. Building Health networks and standardization of clinical processes are the main trend facing these challenges. Actually, the main instrument for documentation and managing of clinical processes are Clinical Pathways (CPs). They allow to transfer evident knowledge in practice and they are the central point of clinical quality management. In contrast, Clinical Practice Guidelines (CPGs) aggregate the evident medical knowledge in a compact form to support physicians in their practice. In the paper analyze the model-driven development of $\mathrm{CP}$ for a health network. Therefore, we work out problems of integration. Finally, the paper shows aspect for a model-based integration of CPGs in CPs. These findings are proofed with the help of the documentation of the regional stroke-network called SOS-NET.
\end{abstract}

Keywords: Process Model, Clinical Practice Guidelines, Clinical Pathways.

\section{Introduction}

Hospitals in Europe especially in Germany are exposed a more competitive business environment as a result of variegated political- and judicial changes. Furthermore the modern media like internet-technologies promote the emancipation of patients. It enables a more competitive situation between hospitals. So, the objectives of care providers concentrate increasingly on the assurance of efficient care on a high level of quality. Consequently, more and more modern economical concepts like process orientated business structures and management are applied in health care sectors.

In this context CPs were established as an instrument for the standardization of clinical business and care processes [1]. Furthermore CPGs were developed by medical associations to aggregate evident knowledge and best practice [2]. CPGs are used to reduce inappropriate variations in practice and to promote the delivery of high quality, evidence-based health care [3].

Different approaches for integration of the content of CPGs in CPs exist (section 4). One of them is the reference model-based approach. Reference models (RM) are special kinds of conceptual models. Conceptual models can be understood as the result of 
a construction "... done by a modeler, who examines the elements of a system for a specific purpose..." [4]. In the information systems discipline, models are used to redesign organizations or to develop software systems $[5,6]$. Therefore, graphical models have been established as "...a medium to foster communication with prospective users..." [7]. RMs are used to communicate best practice, common practice or normative rules [8] (chapter 3).

The present study exhibits the basic idea of the development of RMs, which link CPGs and CPs. Problems are identified with the help of case study, which arise for the RM-based approach. For that case scenarios serve the CPs of the regionally stroke network (SOS-NET), the internal CPGs of SOS-NET as well as the external CPGs of "European Stroke Organization" (ESO) and of the "Deutsche Gesellschaft für Neurologie" (DSG).

The motivation of our research is to analyze methodical ways of integration $\mathrm{CP}$ based on the CPG with the use of RM. The motivation of that paper follows the state of Campbell et al. who characterize as one aim of CPs as: "They can provide a link between the establishment of clinical guidelines and their use." [9]. Two scenarios should clarify aspects of connection of CPs to CPGs.

Scenario I: Implementation of CPs is a huge organizational process. To guarantee a high quality care they need to be developed systematically. A derivation of CP from the CPG would help to reduce manual work. There is always an element of risk associate with such manual procedures.

Scenario II: Since CPs guide evident clinical care, they need to be on the top of medical state of the art. Kaveh et al. showed in their study that CPG are permanent under revision [10]. Due to no real integration especially the alignment of models as well as other CP documents is a complex manual task. Goals for integration are making the process of alignment more efficient. It would also be helpful to check existing CPs due to their compliance.

The present paper dealing with the research question (RQ):

RQ 1 What are the upcoming problems during the development of $\mathrm{CP}$ for the network based on the CPGs?

The study is organized in 6 sections. After case scenario was introduced in section 1.1, the concept of CP and of CPG are introduced based on the literature mentioned in section 2. In section 3 we analyze the status quo of the CPG-integration in CP. In this section we build the fundamentals for the following analysis. In section 4 the concepts of CP and of CPG are compared to identify similarities, which would help to integrate them. In section 5 the results of the comparison are used to indentify issues, which challenge the RM-based approach. The paper ends with conclusions and suggestions for further research (section 6).

\subsection{Project Background - The SOS-Net}

In industrialized countries is the stroke the third major frequent cause of death and the most frequent reason of lasting physical handicaps [11]. In the acute stroke care the capabilities of therapy worsen with each minute, in which the stroke is not diagnosed and not treated accordingly. Immediately a medical intervention is necessary to avoid consequential damage. 
That effort has to be done especially for the radiological findings. Further characteristics for the acute stroke are short delay for decision-making, limited possibility local transfer and difficulty diagnosable symptoms. In 2007, the SOS-NET was founded to build a telemedical infrastructure for regional stroke care. Goal of the network is to treat all stroke patients independent from of the distance to next stroke expert over the whole region on the same level. Within two years the SOS-NET is growing to an important stroke care in Saxony (Germany). At present, twelve hospitals are part of the network (05/2010).

The different suspension of employees and the differences of the technical infrastructure necessitate a defined responsibilities and process within the network. Therefore the stroke center as core of network allocates CPG for the partners of the network (stroke units). A meta-analysis of 17 high quality studies finds that CPs seem to be effective especially for invasive care like stroke care [1]. The CPGs (internal) are used to transfer best practice to the partners, who implement them in CPs, which are used in clinical every day life. Additionally they serve the definition of works principles of the network.

Figure 1 depicts the state-of-the-art of different documentations of clinical processes like they are in stroke care in general (layer 0 - 1) and in the SOS-NET in detail (layer 2 - 3). Conceptual models are used only in the documents of the layers 0 and 1.

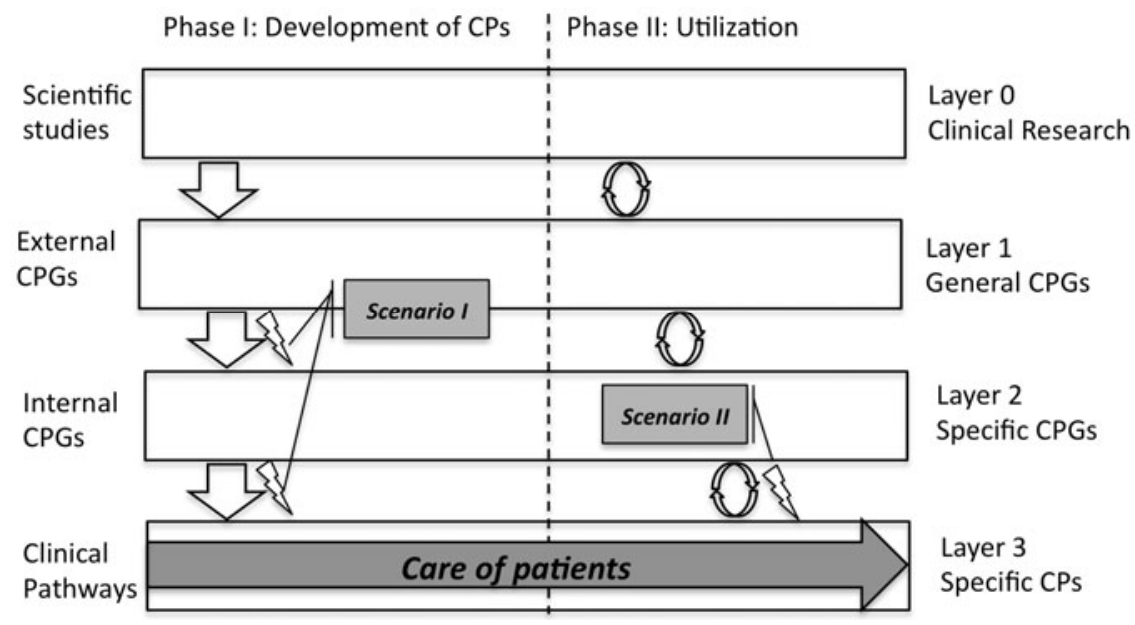

Fig. 1. As-Is-Situation of clinical documentation

\section{Theoretical Foundations}

\subsection{The Clinical Pathway}

CPs are a fundament in process management to achieve economical and qualitative objectives of hospitals [1]. The European Pathway Association defines a CPs as: "Clinical pathways are a methodology for the mutual decision making and organization of care for a well-defined group of patients during a well-defined period. Characteristics of care pathways are: "An explicit statement of the goals and key elements of 
care based on evidence, best practice, and patient expectations; The facilitation of the communication, coordination of roles, and sequencing the activities of the multidisciplinary care team, patients and their relatives; The documentation, monitoring, and evaluation of variances and outcomes; And the identification of the appropriate resources" [12]. The aim of a care pathway is to enhance the quality of care by improving patient outcomes, and promoting patient safety, increasing patient satisfaction, and optimizing the use of resources. The terms Critical Pathway, Clinical Pathway or Integrated Care Pathway are used for this concept synonymously. Some authors try to define them differently $[12,13]$. But until now, no real consensus was found in the scientific community.

\subsection{The Clinical Practice Guideline}

CPGs are defined "as systematically developed statements to assist practitioner and patient decisions about appropriate health care for specific clinical circumstances" [2]. Furthermore, the role of economic statements within the CPG is discussed widely. For example, Priori et al. discuss the impact between economic statements versus the quality of the CPG. They conclude that CPGs should be produced with the primary aim of assessing the quality of science and the strength of evidence of clinical studies [14]. Wollersheim et al. characterize the content and contribution of CPG in the: inclusion of specific recommendations, sufficient supporting evidence, a clear structure and an attractive lay out [15]. The term CPG is used differently and is often not clearly disconnected from related concepts like medical protocol, standard operation procedure or CP. CPG can be developed either locally (internal guidelines) or regionally or nationally (external guidelines) [3]. Hence, several CPGs for same clinical case with different designs and representations exist.

\section{Status Quo of CPG-Integration in Clinical Pathways}

Several methodological approaches for the development of CPs based on the CPGs exist $[13,16-18]$. One approach is to formalize the CPG in a computer-interpretable (CI) form $[19,20]$. Arden-Syntax or XML are markup languages to prepare the narrative CPGs in a CI format. The aim of this approach is to transfer the non-formal rulesystem (clinical algorithm, section 4) of the CPG in decision support systems (DSS) [19]. The DDS supplements the CPs within a hospital [21, 22]. Therefore the hospital information systems (HIS) have to be able to interpret the formal language and combine it to the specific state of patient documented in electronic health record (EHR). The approach of that transformation of CPG in CI structures is thus rather an extension of the paths than a methodology of the systematic transfer and organizational implementation.

A further approach to take CPGs into account of CPs is the inclusion of CPGsanalysis in its research phase during early project stage. Therefore, project members need to analysis the existing literature. This is a highly manual task with no methodical support [20]. The results of the analysis depend on the expertise of the single project member. In order to ensure a high quality of the analysis results several iterations are necessary. 
Another approach, which represents an intermediate way between the formal procedure of CI transformation and not a systematic literature review, is the model-based methodology to transfer CPG-knowledge in CPs. Jacobs et al. present this idea for implementation of CPs in treatment of breast cancer [16]. The central element of their idea is the RM, which was used as instrument to structure and transfer the CPGs content. The purpose of RMs usage is the reduction of development costs and of development time and furthermore the alignment of model construction to common practice or best practice solutions [23]. A further aim is to specify rules and standards that guarantee the compliance to adaptability of a standardized application system or the compliance to organizational rules. The advantage of this approach is that the reference model can be reused. Thus the RM is an adequate instrument around the implementation of CPGs in clinical practice. On the one hand these support the CP creator in the adaptation by construction techniques. On the other hand RMs leave sufficient freedom for the CP-development. Actually, no RM, which includes construction techniques are added to the CPGs. Such construction techniques contain the definition and integration of rules for derivation of specific models. These represent rule sets that define the way of reusing model content and their adaptation and extension [24]. The development of the RM is a cost-intensive process. In contrast are the savings, which can be obtained by the employment of RMs. Due to the multiple applications, in network organization like SOS-NET, RMs can be used to guide best practice for network member. For them conceptual models are taken to communicate best-clinical practice of stroke care. The current models of SOS-NET do not support the adaptation of specific models.

\section{Comparison of Clinical Practice Guideline and Clinical Pathway}

The evaluation criteria that are used based on the concept definition. These are the aim, user groups, type of dissemination, general and specific design characteristics. The major similarity is the approach of each concept. Both purpose a standardization of medical care processes to improve clinical care. In addition to reach a high quality of care, CPs are also focused economic dimension. These derive typically from the formal organizational objectives. CPGs are not as focused on economical aspect as the CPs do [13].

he user groups of the concepts are heterogeneous. The key users of both instruments are the physicians. Patients are the second user group, especially for the CPGs. Additionally, CPs are used by the clinical management and controlling.

Dissemination means the mechanism how clinical knowledge implemented in the clinical practice. CPGs are developed in a general form. That means the physicians have to search actively in databases to CPG. Thus the type of dissemination is passive. CPs are developed in project teams by the care-provider themselves. The process of implementation is a common project step of CPs development. The dissemination of CPs is active. The concepts describe similarity as like as different aspects. CP contain beside clinical content administrative or fosterage services. Moreover, CPs include the entire range of the care process. The level of detail of CPs is on a constant level. These are not selected due to the importance in the care process. The level of abstraction varies according to the importance for the respective medical case. Combinations of prose and tabular form, protocol charts, lists as well as conceptual models 
are used to describe a step-per-step procedure for solving a clinical problem in both concepts. These procedures are defined as clinical algorithm [25]. They are subjected to both concepts like a skeleton. The clinical algorithm is an important similarity, which can help to integrate both concepts.

\section{Issues of Development}

According to the approach of Jacobs et al. the present analysis (scenario I) follows based on the main steps: Preparation of the CP project, Modeling of the CPGs, if it were not made with the CPGs development, Implementation of construction techniques (placeholders, configuration and construction advises) of RMs, Adaptation of $\mathrm{RM}$ by the specification of RM to a CP model, Dissemination of CP within the hospital. The task of preparation phase is the determination of CPGs, which are associated for the CP. The relation between CPGs and CP is not similar and is not defined by any institution. The relation between sections of the $\mathrm{CP}$ and of the CPGs has to be organized. In case of the SOS-NET, we have five relevant CPGs, which need to be taken in account. Different recommendations, which have to be integrated, exist. Thus it is required to have modular components, which compose these recommendations. The next step (step 2) of the procedure can be skipped, if the CPG as conceptual model exists already, and if they have the same format. Otherwise, the clinical algorithm of the guideline has to be extracted and modeled. The possible languages are Standard Nomenclature of MDM [25], Flowchart or Business Process Modeling Notation (BPMN) or Activity Chart of Unified Modeling Language (UML).

Due to the multiple relations between the concepts different CPGs have to be considered for CP. The kind of the composition cannot be pre-defined, since they find in each case in differently contexts application. Consequently, integrations of the conceptual models are necessary. CPGs lay on level of abstraction layer. The so-called problem of horizontal integration is mentioned in previous literature [26]. If it is necessary to model the CPGs again, additionally an integrated model of the CPG has to be developed. Otherwise the different languages have to be adapted. In the third step, the model of the CPGs is transferred a reference model. Up-to-date no standardized reference model language is accepted. Exemplarily, the ARIS method (architecture of integrated information systems) often used the event-controlled process chain (EPC) as well as the entity relationship model (ERM) [27]. In stroke care external CPGs are in prose. The solution of the SOS-NET is to describe the CPG with the help of Flowcharts. In addition, migration schemata, which allow transforming the flowchart to another type of process model, are published. We used the Flowchart because it is widely accepted, easily to understand. To enrich the clinical content of the CPG with administrative process aspects we add to the CPG model additional content of the stroke center. Furthermore, we implement placeholder at position, which need to specify while adaptation.

In the fourth step, the RM is adapted to a specific model. Therefore the path team implements the CPs with help of the construction techniques. An important fact, which has to be taken into account, is that if the goal modeling language of the $\mathrm{CP}$ deviates itself from the reference model language, the adapted model has to migrate in the right modeling language. In contrast to CPGs, CPs describe all activities of clinical care. For the adaptation of $\mathrm{CP}$ that means that additional aspect like administrative 
processes need to be create. Due to the indispensable fact that the CPGs are more general and the CPs are more detailed no aspects are found.

\section{Conclusion and Further Research}

In that paper the different concepts of CPG and CP are presented. With the method of case study the situation in SOS-NET was analyzed. The CPG and CP were compared on a conceptual level and similarities and differences were observed. The comparison of the CPGs and CPs shows that the clinical algorithm is an important similarity, which could be used to link the concepts. In the state-of-the-art we showed that the RM-based approach is one possible way to integrate both concepts. The identified problems are the relation between the concepts, the different aspects and the not standardized design. Furthermore the RM as technique itself raises problems like the missing tool support and evaluated RM-languages. Finally, the study shows that a methodical RM-based development of CP based on CPG can be built. One of the main deficits is the missing standardization for design of CPGs and CPs. Especially, CPGs should be published in combination with a RM and a description how to use it. That would be help to assure a constant quality of dissemination. Further research is need on detailed method of implementation and on a method for utilization and for half-automatic alignment of CP. Moreover, the alignment as instrument of clinical process quality should be investigated. An empirical validation of the case study results is also necessary.

\section{References}

1. Rotter, T., Kugler, J., Koch, R., Gothe, H., Twork, S., van Oostrum, J., Steyerberg, E.: A systematic review and meta-analysis of the effects of clinical pathways on length of stay, hospital costs and patient outcomes. BMC Health Services Research 8, 265 (2008)

2. Field, M.J., Lohr, K.N.: Clinical practice guidelines: directions for a new program. National Academies Press, Washington (1990)

3. Thomas, L.: Clinical practice guidelines. Evidence Based Nursing 2, 38-39 (1999)

4. Schuette, R., Rotthowe, T.: The Guidelines of Modeling - An Approach to Enhance the Quality in Information Models. In: Ling, T.-W., Ram, S., Li Lee, M. (eds.) ER 1998. LNCS, vol. 1507, pp. 240-254. Springer, Heidelberg (1998)

5. Fettke, P., Loos, P.: Klassifikation von informationsmodellen-nutzenpotenziale, methode und anwendung am beispiel von referenzmodellen. In: Working Paper of the Research Group Information Systems \& Management, Universität Mainz, vol. 9 (2002)

6. Rosemann, M., Schwegmann, A., Delfmann, P.: Vorbereitung der Prozessmodellierung. In: Prozessmanagement, pp. S45-S103. Springer, Berlin (2002)

7. Frank, U.: Conceptual modelling as the core of the information systems disciplineperspectives and epistemological challenges. In: Proceedings of the Fifth Americas Conference on Information Systems (AMCIS 1999), pp. S13-S15 (August 1999)

8. Becker, J., Delfmann, P., Ralf, K.: Konstruktion von Referenzmodellierungssprachen - Ein Ordnungsrahmen zur Spezifikation von Adaptionsmechanismen für Informationsmodelle, pp. 251-264 (2004)

9. Campbell, H., Hotchkiss, R., Bradshaw, N., Porteous, M.: Integrated care pathways. BMJ, 133-137 (1998) 
10. Kaveh, D., Shojania, M., Ansari, M., Ji, J., Doucette, S., Moher, D.: How quickly do systematic reviews go out of date? A Survival Analysis. Anal. of Internal Medicine 147, 224 233 (2007)

11. Kolominsky-R., P.L., Heuschmann, P.U., Marschall, D., Emmert, M., Baltzer, N.: Lifetime Cost of Ischemic Stroke in Germany: Results and National Projections From a PopulationBased Stroke Registry. Stroke 37, 1179-1183 (2006)

12. De Bleser, L., Depreitere, R., De Waele, K., Vanhaecht, K., Vlayen, J., Sermeus, W.: Defining pathways. Journal of Nursing Management 14, 553-563 (2006)

13. Greiling, M., Rudloff, B.: Klinische Pfade optimal gestalten. Baumann Fachzeitschriften Verlag, Berlin (2005)

14. Priori, S.G., Klein, W., Bassand, J.P.: Medical Practice Guidelines: separating science from economics. European heart journal 24, 1962 (2003)

15. Wollersheim, H., Burgers, J., Grol, R.: Clinical guidelines to improve patient care. The Netherlands Journal of Medicine 63, 188-192 (2005)

16. Jacobs, B., Oberhoff, C., Stausberg, J.: Ableitung von Klinischen Pfaden aus evidenzbasierten Leitlinien am Beispiel der Behandlung des Mammakarzinoms der Frau, http: / / www. egms. de/static/de/journals/mibe/20073/mibe000057.shtml

17. Hellmann, W.: Praxis Klinischer Pfade: Viele Wege führen zum Ziel. Ecomed. (2003).

18. Schluechter, D., Conen, T., Holler, T., Müller, H., Schmid, K.: Pfad-Controlling im Modell integrierter Patientenpfade - ein neues, innovatives Prozesscontrolling-Instrument. Gesundheitsökonomie \& Qualitätsmanagement 8, 355-362 (2003)

19. Peleg, M., Tu, S., Bury, J., Ciccarese, P., Fox, J., Greenes, R.A., Hall, R., Johnson, P.D.: Comparing Computer-interpretable Guideline Models: A Case-study Approach. Journal of the American Medical Informatics Association 10, 52-68 (2003)

20. Kaiser, K., Akkaya, C., Miksch, S.: How can information extraction ease formalizing treatment processes in clinical practice guidelines? Artif. Intell. Med. 39, 151-163 (2007)

21. Arden Syntax, http: / /www.hl7.org/implement/standards/ardensyntax.cfm

22. ASTM E2210 -06 Standard Specification for Guideline Elements Model version 2, http: / / www . astm.org/Standards/E2210.htm

23. Schütte, R.: Grundsätze ordnungsmäßiger Referenzmodellierung. Gabler (1998)

24. vom Brocke, J., Buddendick, C.: Referenzmodellierung: Grundlagen, Techniken und domänenbezogene Anwendung. Physica-Verlag, Heidelberg (2007)

25. Barak, N., Margolis, C., Gottlieb, L.: Proposal for Clinical Algorithm Standards: Society for Medical Decision Making Committee on Standardization of Clinical Algorithms. Medical Decision Making 12, 149-154 (1992)

26. Rosemann, M.: Komplexitätsmanagement in Prozeßmodellen. Gabler, Wiesbaden (2002)

27. Scheer, A.: ARIS-business process frameworks. Springer, Heidelberg (1999) 